\title{
A source to deliver mesoscopic particles for laser plasma studies
}

R. Gopal, R. Kumar, M. Anand, A. Kulkarni, D. P. Singh, S. R. Krishnan, V. Sharma, and M. Krishnamurthy

Citation: Rev. Sci. Instrum. 88, 023301 (2017); doi: 10.1063/1.4974973

View online: http://dx.doi.org/10.1063/1.4974973

View Table of Contents: http://aip.scitation.org/toc/rsi/88/2

Published by the American Institute of Physics

STEM CAREER

on networking, interviewing, conferences, presenting...

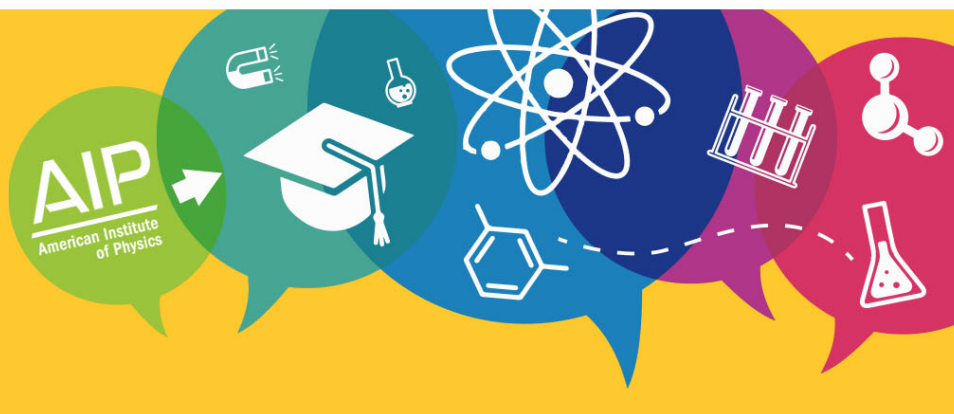




\title{
A source to deliver mesoscopic particles for laser plasma studies
}

\author{
R. Gopal, ${ }^{1}$ R. Kumar, ${ }^{2}$ M. Anand,${ }^{1}$ A. Kulkarni, ${ }^{1}$ D. P. Singh, ${ }^{1}$ S. R. Krishnan, ${ }^{3}$ V. Sharma,${ }^{2}$ \\ and M. Krishnamurthy ${ }^{1, a)}$ \\ ${ }^{1}$ Tata Institute of Fundamental Research, 21, Brundhavan Colony, Hyderabad 500075, India \\ ${ }^{2}$ Department of Physics, Indian Institute of Technology Hyderabad, Kandi, Sangareddy 502285, India \\ ${ }^{3}$ Department of Physics, Indian Institute of Technology Madras, Chennai 600036, India
}

(Received 20 October 2016; accepted 11 January 2017; published online 6 February 2017)

\begin{abstract}
Intense ultrashort laser produced plasmas are a source for high brightness, short burst of X-rays, electrons, and high energy ions. Laser energy absorption and its disbursement strongly depend on the laser parameters and also on the initial size and shape of the target. The ability to change the shape, size, and material composition of the matter that absorbs light is of paramount importance not only from a fundamental physics point of view but also for potentially developing laser plasma sources tailored for specific applications. The idea of preparing mesoscopic particles of desired size/shape and suspending them in vacuum for laser plasma acceleration is a sparsely explored domain. In the following report we outline the development of a delivery mechanism of microparticles into an effusive jet in vacuum for laser plasma studies. We characterise the device in terms of particle density, particle size distribution, and duration of operation under conditions suitable for laser plasma studies. We also present the first results of x-ray emission from micro crystals of boric acid that extends to $100 \mathrm{keV}$ even under relatively mild intensities of $10^{16} \mathrm{~W} / \mathrm{cm}^{2}$. Published by AIP Publishing. [http://dx.doi.org/10.1063/1.4974973]
\end{abstract}

\section{INTRODUCTION}

Femtosecond laser interaction with matter at intensities above $10^{14} \mathrm{~W} / \mathrm{cm}^{2}$ inevitably proceeds through the rapid ionization of constituent atoms leading to the formation of plasma on time scales of a few tens of femtoseconds, often times even before the peak of the pulse has arrived. ${ }^{1,2}$ In short pulse lasers of $\leq 100 \mathrm{fs}$ duration, ion motion is negligible, implying that the laser directly transfers energy to the electrons in the plasma. Further laser absorption, plasma evolution, and energy disbursement strongly depend on the initial state of the matter that receives the intense laser pulses. For example, when a flat slab of solid is used as a target, a critical density plasma sheath is quickly formed in the initial part of the pulse that reflects most of the remaining pulse and reduces the absorption. ${ }^{3}$ Nanoparticles disbursed on a solid substrate increase the efficiency of hot electron generation and x-ray emission. ${ }^{4}$ With isolated nano-clusters (of size smaller than laser wavelength) the laser absorption is efficient ${ }^{5}$ (more than $90 \%$ ) and most of the energy is expended in atomic ionisation ${ }^{6}$ followed by ion acceleration. The ability to absorb all the incident energy and use it to strip nearly all the electrons of all the atoms in a nm deuterium cluster has even led to the possibility of observing efficient nuclear fusion. $^{7}$

The question of scaling this to even larger systems and possibility of controlling the plasma evolution through the changes in the initial size, shape, and material composition is quite open. ${ }^{8}$ For example, in the query about material composition, it was observed that a minuscule doping of Xe in helium clusters increased the laser absorption, ${ }^{9}$ while similar increases in electron emission and X-ray emission ${ }^{10}$ were observed with

\footnotetext{
a) mkrism@tifr.res.in. URL: www.tifr.res.in/ uphill.
}

replacement of about 5\% of water molecules in an argon cluster. Addition of 4-5 Xe atoms in a He cluster dramatically changes the ionisation of the cluster. ${ }^{11}$ With reference to size and shape dependencies, hard X-rays, which are an indication of the electron temperature, have been shown to have a near 20 -fold enhancement from plasmas created on surfaces with nanometric modifications $\mathrm{s}^{4,12}$ as compared to those on polished surfaces. As revealed by particle-in-cell plasma simulations, ${ }^{12}$ depending on the aspect ratio, length, and density of the nanostructures on the surface, the enhancement in the intensities can be up to 100 fold. Increasing the size to $1-2 \mu \mathrm{m}$ and using the ellipsoidal shaped E. coli bacteria, a large enhancement of two orders in magnitude in the X-ray yield has been observed with respect to polished substrates. When these bacteria are doped with nanoparticles, ${ }^{13}$ the enhancement in X-ray yield is 10000 fold! The intriguing physics behind the enhanced efficiency is complicated as the bulk plasma profiles are modified and the modification in the anharmonicities in the electrostatic potentials over and above the bulk plasma needs to be deciphered. ${ }^{14}$ To be noted is that these studies with mesoscopic particles have been limited to surface modifications and not to true isolated mass limited targets. In isolated systems the size/shape effects of the nano structures can be expected to not be overshadowed by bulk plasma effects. Furthermore the reduction in available surface area of isolated mass-limited targets leads to higher charge densities and a better spatial confinement of the electron cloud as well as recirculation of hot electrons. While local field enhancements at the surface of the targets which can be attributed to Mie focussing produce higher electron densities, penetration of surface plasma waves into the interior also increases the ionisation within the target. ${ }^{15}$ The predicted enhancement leading to large hot-electron temperatures has already been shown in plasmas created in micron-scale liquid droplets. ${ }^{16,17}$ 
A systematic study of how the shape and size of the nanoor micro-particles changes the laser matter interaction and to modify, say, the energy disbursement in the mode of X-rays has not been effectively explored. Supersonic jet methods, where the clusters are made from atomic or molecular system by aggregation, seldom produce clusters that are not spherical. Even in methods where mesoscopic particles are made from bulk, in liquid droplet experiments, for example, surface tension drives the system to a spherical drop. Should we engineer targets which can combine any desired features of micrometer spatial confinement with nanometric sub structuring, we may pose questions on the length scaling of electron heating processes from nanometeric to micrometric scales, with implications to applications such as particle acceleration and high energy photon generation. A method to introduce such particles prepared with any desired shape, size, and material composition into the laser focus volume is then pertinent to address some of these core issues of intense laser plasma physics.

In this submission we have considered a novel approach to engineer such targets for laser plasma studies. We report on the development of a mechanism to deliver sub-micron to micron size particles as an effusive, but spatially well-confined jet, into vacuum. The method is general and can be used with any particle that is pre-synthesised in any shape to include all the conceivable sub-structuring, thereby allowing structure dependent studies on isolated targets for the first time. Additionally, the approach offers an effective way of replenishing solid targets, particularly important in the context of current developments in high-repetition lasers needed to translate ion/electron acceleration schemes to real-world applications. The strategy employed here is akin to particle seeding used in particle image velocimetry. ${ }^{18}$ Pressurised gas passing through a bed of the pre-synthesised particles forms an aerosol mixture of the particles in the gas. The jet of particles carried by the gas then interacts with the focussed laser beam in vacuum. We describe the design of such a particle delivery mechanism and characterise the consistency of operation of the device, the particle density delivered at the laser focus, and particle size distribution (for a powder with varying particle sizes). We also present the first results on the x-ray emission from microcrystalline boric acid particles using a multi-millijoule, kilohertz femtosecond laser.

\section{PARTICLE SEEDER}

Seeding particles into a gas stream can be effected either by pickup through "cyclone"-type turbulences or alternatively by "bubbling" the carrier gas through a fluidized bed of particles. ${ }^{18}$ In Figs. 1(a) and 1(b) are sketches of two devices fabricated for entrainment of particles into a carrier gas, which use the bubbling and cyclone mechanisms, respectively. For the cyclone type design, compressed gas is introduced into the cylindrical powder reservoir (diameter: $35 \mathrm{~mm}$, length: 100 $\mathrm{mm}$ ) as shown in Fig. 1(b) through a central tube. The exit of the tube is constricted to a $1 \mathrm{~mm}$ orifice. The flow of the gas is interrupted by a baffle which directs the gas onto the powder. The eddies in the chamber gas lift particles from the powder surface into the gas, which are carried to the top of the chamber, which is flanged with a standard CF 35 vacuum flange. A short tube at the exit of the chamber carries the aerosol into a vacuum chamber.

For the bubbling type seeder Fig. 1(a), the gas is introduced from the side. In typical designs a gas distributor is included to provide uniform gas distribution profile under the bed of particles. At a minimum gas flow $u_{m f}$, referred to as the minimum fluidization velocity, the drag force on the particles is commensurate with the gravitational force. A reaction force on the fluid by the particle bed causes a pressure drop across the bed $\Delta P_{\text {bed }}$. Simple force balancing (ignoring friction from the wall surfaces) yields ${ }^{19}$

$$
\Delta P_{\text {bed }}=g H_{\text {bed }}\left(\rho_{p}-\rho_{g}\right)(1-\epsilon) .
$$

Here $H_{b e d}$ is the height of the particle bed, $g$ is the acceleration due to gravity, $\rho_{p}$ is the particle density, $\epsilon$ is the void fraction in the powder bed, and $\rho_{g}\left(\ll \rho_{p}\right)$ is gas density.

The particles entrained in the aerosol follow the flow lines into the interaction vacuum chamber shown in Fig. 1(a). To visualise the consistency in particle density over time, a $650 \mathrm{~nm}$ $\mathrm{CW}$ laser beam was passed through the particle jet as shown in Fig. 2. Scattered light was recorded over time by a photodiode $(\mathrm{PD})$ at a very small angle $\approx 5^{\circ}$ to the incident beam, along the direction of propagation. In Fig. 1(c), the photodiode signal is plotted as a function of time in minutes. The particle bed is filled with $40 \mathrm{gm}$ of commercial grade polycrystalline boric acid $\left(\mathrm{H}_{3} \mathrm{BO}_{3}\right.$ ) powder (bulk density $\left.=0.7 \mathrm{gm} / \mathrm{cm}^{3}\right)$. Assuming that the particle size distribution does not change as a function of time, the photodiode signal is directly proportional to particle density at the interaction zone. The black line therefore plots the particle density when the fluidisation of the particle is realised only by aerodynamic means, i.e., bubbling alone. A very sharp rise of the measured signal to its peak at the beginning when gas is introduced into the chamber, followed by a slow fall in the signal over 5 min to a constant settled level, however punctuated by rapid bursts of fluctuations. The settled level is low enough to consider it as the base signal where no particles are observed. Similar behaviour is seen when the entrainment is brought about in a cyclone type system (as in Fig. 1(b)). The major limitation of these seeding designs when seen in the context of usage of the particle beam for laser plasma studies is the very short duration of operation of only around $5 \mathrm{~min}$. This is a major drawback, particularly when it is required to build statistics in charged particle and photon spectra from the laser plasmas over a large number of laser shots.

A seminal classification of powders fluidized by gases which can provide criteria to generally predict particle behaviour in a fluidized bed is provided by Geldart. ${ }^{20} \mathrm{Based}$ on the Sauter mean diameter (diameter of a sphere with same volume/surface area ratio), $d_{p}$, and the particle density, particles can be classified into four so-called Geldart groups: A, B, C, and D. Group A particles with $d_{p}$ in the range $20 \mu \mathrm{m}-125 \mu \mathrm{m}$ and densities smaller than $1.5 \mathrm{gm} / \mathrm{cm}^{3}$ are smoothly fluidizable, with low bubble formation. Large particles $\left(1.5-4 \mathrm{gm} / \mathrm{cm}^{3}, d_{p}=150-1000 \mu \mathrm{m}\right.$ : group B; $d_{p} \geq 1000 \mu \mathrm{m}$ : group D) allow the formation of large bubbles. They are relatively resistant to fluidisation as compared to group A 


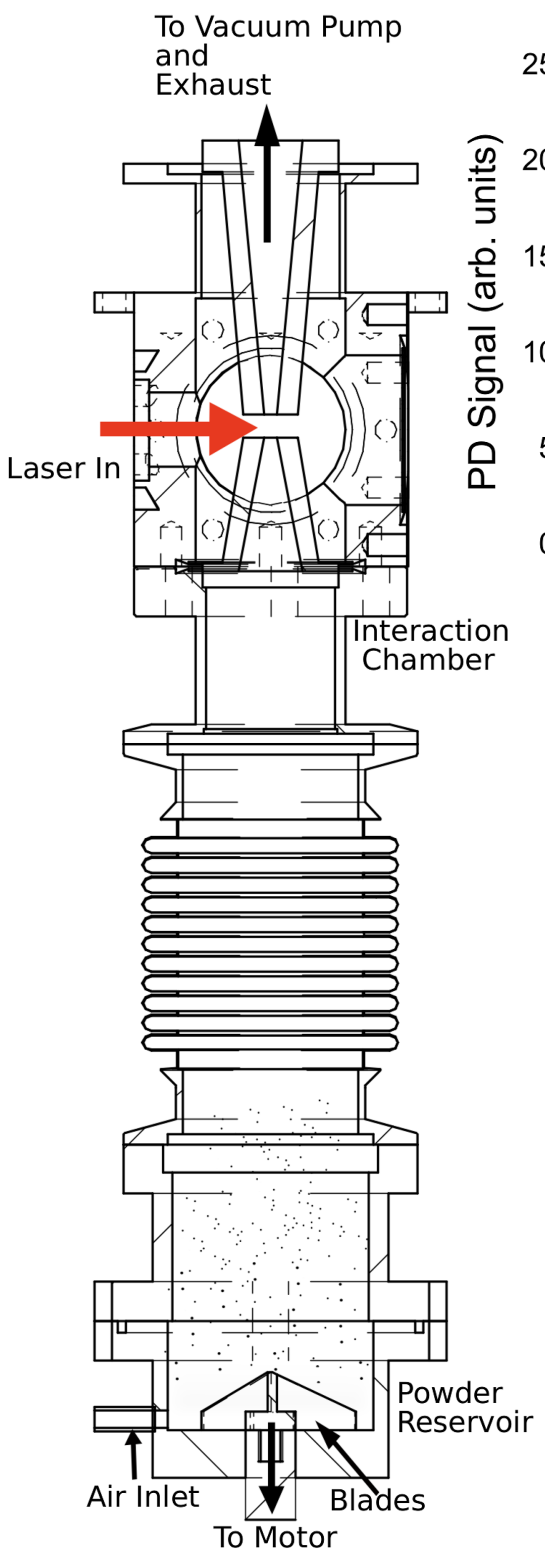

(a)
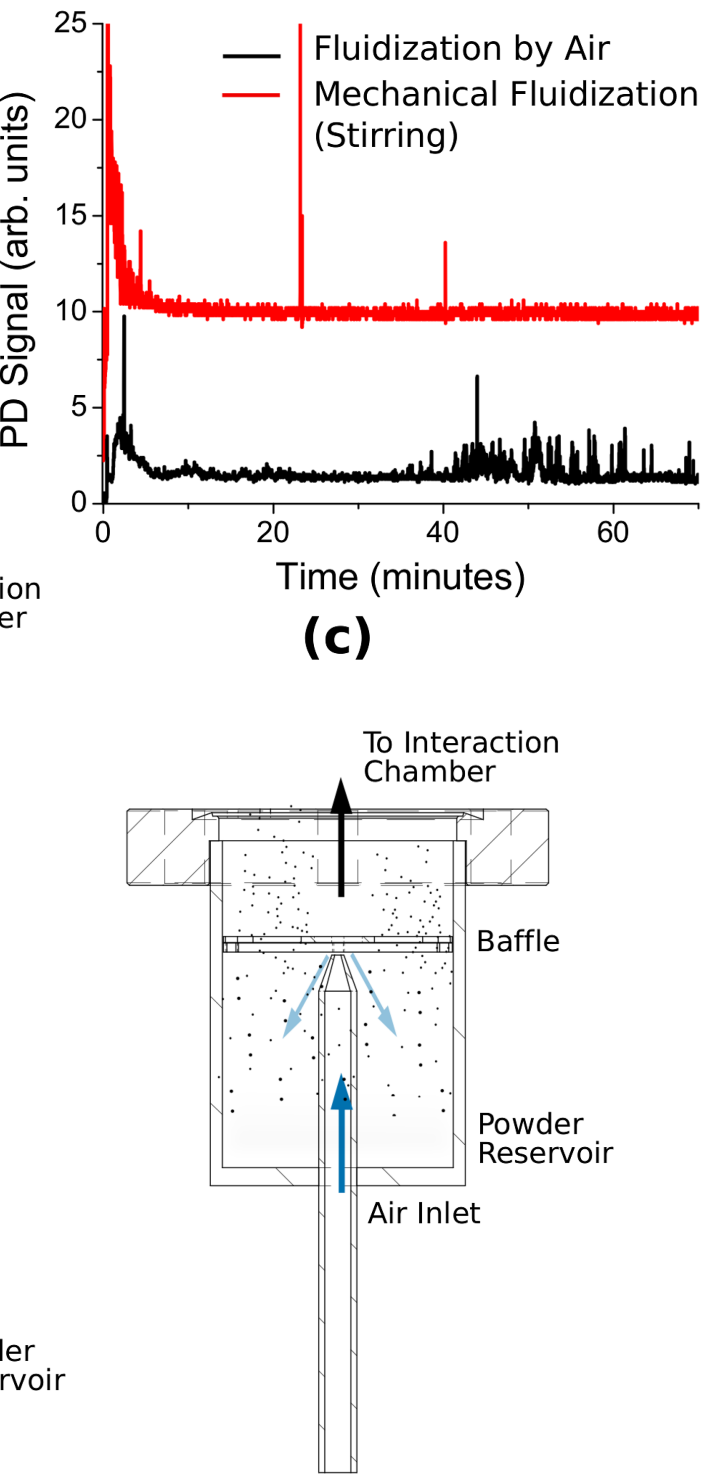

FIG. 1. (a) Sketch of the particle seeding machine which uses "bubbling" to fluidize the particles. (b) A prototype machine for seeding using "cyclone"type entrainment. (c) Photodiode (PD) signal from scattering of $650 \mathrm{~nm}, \mathrm{CW}$ laser beam at $5^{\circ}$ to propagation direction of the laser beam. Black curve corresponds to the time-decay of the signal when fluidisation is only by air (see text). In red, the curve shows the temporal behaviour of the signal when mechanical fluidisation is introduced. The signals vary a lot in the initial period when the carrier gas is introduced and then reaches a steady state. The X-ray measurements (in Fig. 4) were taken at the time the system had stabilized, typically around 5 minutes after the flow of the carrier gas was started. particles. Particles less than $30 \mu \mathrm{m}$ in size (group C) are most difficult to fluidize. They are cohesive and therefore, the inter particle forces dominate over the drag forces of the carrier gas. Gas passing through the distributors lifts the powder bed entirely leading to the formation of "rat-holes" or channels. However, for setting up mesoscopic laser plasmas, the particle sizes required would be in the range of $0.1-10 \mu \mathrm{m}$.

Essentially, an alternate system is required to ensure "fluidity" of the bulk of the powder. We therefore introduced a rotating blade (Fig. 1(a)) driven by an external motor (100 rpm) which would prevent gas channels from forming in the bulk of the powder. In Fig. 1(c), the red curve indicates the scattered photodiode signal as a function of time for particles fluidized by mechanical means. In comparison on an absolute scale to the aerodynamically fluidized time signal, it is clear that the particle density has increased 5-6 fold. The base levels of the signals (black and red lines) in Fig. 1(c) are same. So the enhanced scattered signal in red line shows a direct measure of the higher particle density operational sustained over a longer time period of operation. The settled signal level is $40 \%$ of the peak value that remains constant over an hour. Additionally, consistency of the particle density over time in the settled period is very good with a standard deviation of $2 \%$ of the median. Since inter-particle cohesive forces are dominant in group $\mathrm{C}$ powders, an indication of satisfactory fluidization is the absence of particle aggregates ${ }^{21}$ in the aerosol. Elutriation by gravitational means, which is simply realised by having a vertical long chamber, also chooses smaller, non-agglomerated particles. To verify that agglomeration is negligible for the particle seeder reported here, we set up an imaging system. As shown in the schematic in Fig. 2, the scattering from the particles is imaged through a zoom lens (VZM 450, Edmund Optics) at $3 \times$ onto a high speed ( $15 \mathrm{fps}$ at $1392 \times 1040$ pixels $)$ CCD camera (Lu135, Lumenera Corporation) at $90^{\circ}$ to the 


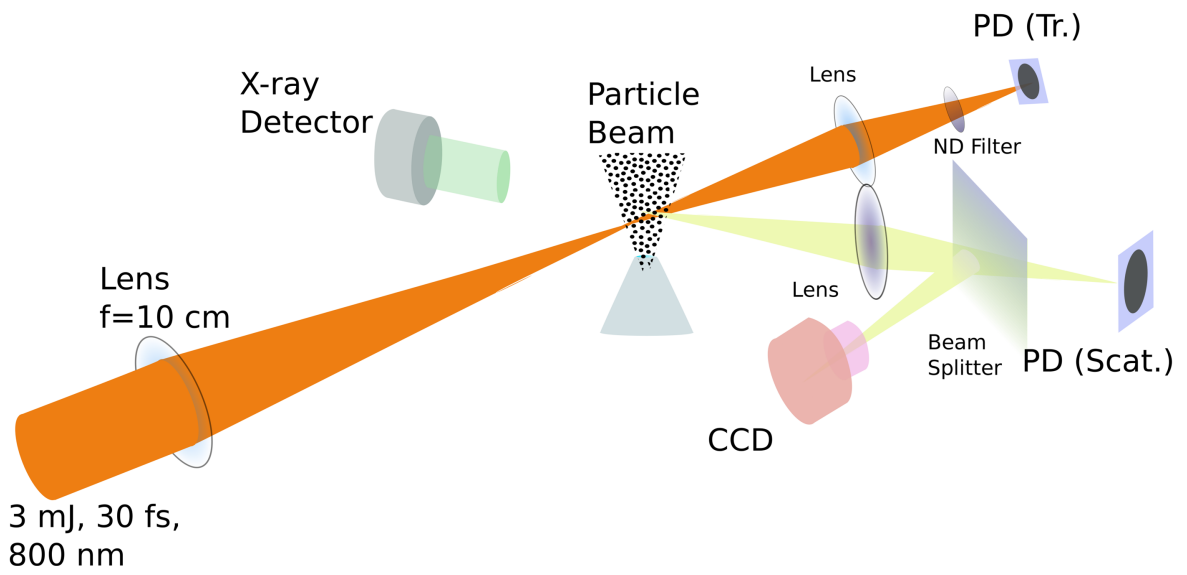

FIG. 2. A schematic representation of the experimental setup for generation of laser plasmas from micro particles and associated diagnostics. The same setup was used also for visualisation of particles in the interaction region to estimate particle size distribution and density, using the CCD camera. The laser power was reduced to less than $1 \mathrm{~mW}$ for these measurements. An X-ray detector was placed at $90^{\circ}$ to the laser propagation. A fast photodiode (PD Scat.) captured the scattered light at $90^{\circ}$, through a zoom lens. The transmitted light was collected by a $f=75 \mathrm{~mm}$ lens onto another fast $800 \mathrm{~nm}$ photodiode (PD Tr.).

incident $30 \mathrm{fs}, 1 \mathrm{kHz}$ femtosecond laser (Femtopower V, Femtolasers $\mathrm{GmBH}$ ) beam. For the imaging experiments, a low power $(\sim 1 \mathrm{~mW})$ beam was focussed onto the particle beam at the centre of the vacuum chamber. Multiple snapshots (1000) were captured and analysed by ImageJ software to count and sort the particles as per their sizes. The particle size distribution thus obtained is plotted in Fig. 3(a). From the average number of particles per frame, we can estimate the particle density to be $\sim 1 \times 10^{6}$ particles $/ \mathrm{cm}^{3}$. The average particle size is estimated to be $14 \mu \mathrm{m}$. A smooth exponential fall in the particle size distribution should be noted indicating decreasing tendency for heavier particles to be transported to the interaction region. Inspection of the images also did not reveal any particle aggregates at the interaction region.
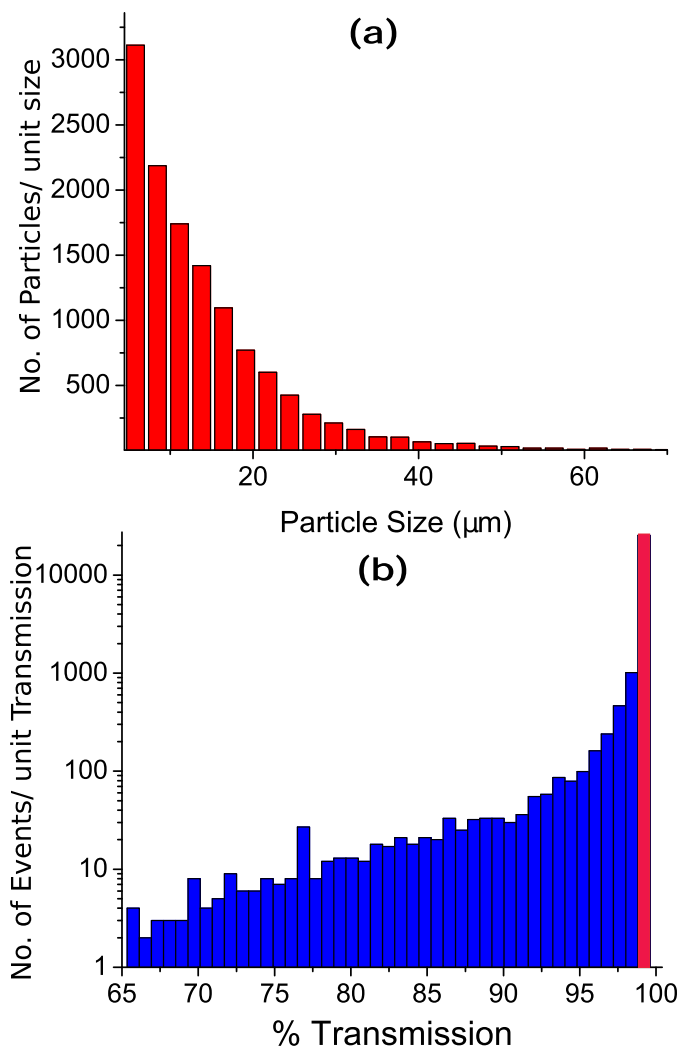

FIG. 3. (a) The particle size distribution obtained by analysing the images of the scattering by particles. (b) A histogram of photodiode voltages observed on the transmission photodiode (PD Trans., Fig. 2).

\section{FIRST RESULTS FROM SIZE-LIMITED MESOSCOPIC LASER PLASMAS}

Having shown an adequate advancement in stable operation of the delivery mechanism for extended durations, we proceed to report here the first experiments to study the interaction of the isolated mass-limited solid microparticles with intense femtosecond laser pulses. The experimental setup is schematically shown in Fig. 2. The particles are introduced into the centre of the vacuum chamber through a converging nozzle with an exit diameter of $3 \mathrm{~mm}$. The particle beam is intercepted at a height of around $6 \mathrm{~mm}$ by an expanding cone with an input diameter of $6 \mathrm{~mm}$ as shown in Fig. 1(a). This acts as a receptacle for the particles minimizing the contamination of surfaces with fine particle dust, particularly, the entrance and exit window for the laser, and the observation window for scattering at $90^{\circ}$. The receiving chamber after the expanding cone also acts as a trap for the particles (for recycling the powder and to prevent contamination of the vacuum pump). A rotary pump evacuated the vacuum chamber to base pressure conditions of 0.05 mbar. Under typical experiment conditions, a flow rate of $1.5 \mathrm{mbar} 1 / \mathrm{s}$ was maintained, with the operating pressure in the interaction chamber at 0.5 mbar.

The $800 \mathrm{~nm}$ laser pulses of $30 \mathrm{fs}$ pulse duration and a pulse energy of $2.7 \mathrm{~mJ}$ are focused in a $\mathrm{f} / 5$ geometry into the boric acid particle beam, to a measured $1 / e^{2}$ beam diameter of $\sim 25 \mu \mathrm{m}$. This results in a moderate peak intensity $\left(I_{0}\right)$ of $4 \times 10^{16} \mathrm{~W} / \mathrm{cm}^{2}$. The focal volume is computed to be $1 \times 10^{-7} \mathrm{~cm}^{3}$. Therefore, from the particle density the probability of finding a particle in the laser focal volume comes around to $10( \pm 1) \%$. We acquired the scattered light and the transmitted light pulses on fast photodiodes (PD's), shot-toshot triggered by the input laser pulse on a fast oscilloscope. A LabVIEW module was implemented to acquire the peak to peak values of each of the signals from the oscilloscope. These measurements were taken at the time the system had stabilized, typically around $5 \mathrm{~min}$ after the flow of the carrier gas was started.

A particle in the focal volume absorbs energy through nonlinear mechanisms, ${ }^{1,2,5}$ which is evidenced by a decrease in the transmitted laser energy after the interaction. (The transmission can also be reduced due to scattering by the particle.) Therefore a decrease in the transmitted photodiode 
pulse height indicates an interaction event. A histogram of the recorded shot-to-shot pulse heights normalised to the maximum pulse height is shown in Fig. 3(b). It is evident that a large number of laser shots do not interact with any particle (red bar in the histogram). From a total of 28159 events, 2758 events can be considered as events where the transmission has fallen. In other words, the probability of interaction is $9( \pm 2) \%$. This corroborates our earlier estimate based on the particle density in the focal volume.

The soft and hard X-ray spectrum from bremsstrahlung of the plasma electrons gives a direct measure of the hot electron temperatures of the transient micro-plasma generated in the intense pulse interaction. The low energy part of the X-ray emission spectrum is measured through a Mylar window by a Si PIN detector (XR-CR 100, Amptek, Inc.) and recorded by an MCA (model 8000, Amptek, Inc.) at a distance of $5 \mathrm{~cm}$ from the interaction region. The hard $\mathrm{X}$-ray spectrum in the range of $30-150 \mathrm{keV}$ is measured by a $\mathrm{NaI}(\mathrm{Tl})$ detector placed at about $150 \mathrm{~mm}$ from the interaction region. The active detector area was reduced to $3 \mathrm{~mm}$ using lead plates, and the detector placed at a distance of $20 \mathrm{~cm}$ to reduce the probability of pile-up effects in the detector. At this configuration we acquired X-rays at a count rate of one in 200 laser shots to completely avoid errors in the electron temperature deciphered from the x-ray spectrum. Furthermore background signal due to cosmic rays, etc. was filtered by acquiring the background spectrum for the same amount of time as the X-rays from the laser plasma. The background subtracted data are presented in Fig. 4, as blue dots. The X-rays pass through $1.5 \mathrm{~mm}$ of a glass window (of the vacuum chamber) and $2 \mathrm{~mm}$ of the aluminium shielding of the detector, before being detected. So we convolved the raw spectrum with the energy transmission functions of these materials, ${ }^{22}$ to obtain the spectrum represented by black squares, which emerges as a typical bremsstrahlung $\mathrm{X}$-ray spectrum. If we assume a Maxwellian distribution of the electrons, with two temperatures $T_{e 1}$ and $T_{e 2}$ in the plasma as

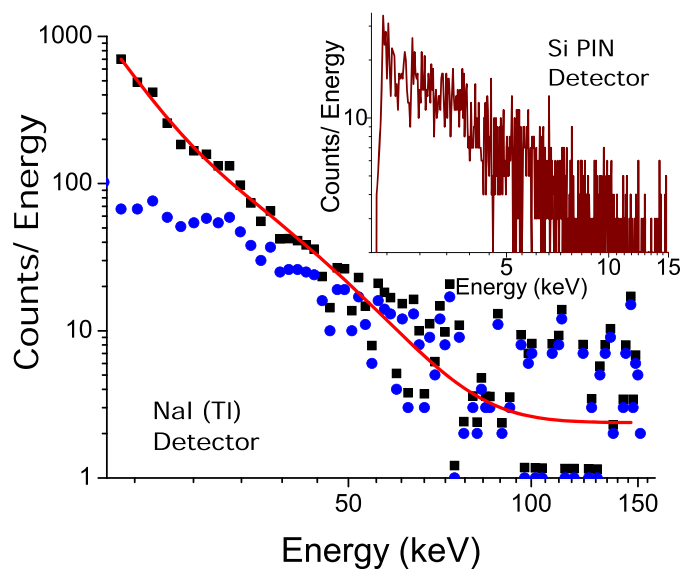

FIG. 4. Hard X-rays from the bremsstrahlung of hot electrons from the laser plasma formed from boric acid particles in the jet measured by a NaI(Tl) Xray detector. The blue dots represent the X-ray spectrum as measured by the detector. The raw spectrum is convoluted with X-ray energy transmission profiles for material in the path to give the spectrum represented by black squares. The red curve is the two-electron temperature fit, assuming a Maxwellian distribution. The inset shows the low energy X-ray spectra captured by a Si PIN detector. the genesis of the X-rays, the bremsstrahlung can be fit to a distribution given by the red curve. A hot electron energy temperature $\left(T_{e 2}\right)$ of $12 \pm 2 \mathrm{keV}$, with a tail extending to $100 \mathrm{keV}$, can be gleaned from the spectrum. $T_{e 1}$ is seen to be $2.8 \pm 0.3 \mathrm{keV}$. The inset shows a typical low energy X-ray spectrum (5-20 keV) obtained by the Si PIN detector. To be noted is that the Si PIN detector efficiency falls away for Xray energies above $15 \mathrm{keV}$, while the $\mathrm{NaI}$ (Tl) detector cannot detect below $20 \mathrm{keV}$. The spectrum in the inset is not corrected to absolute detection efficiencies and solid angles to compare with the high energy spectra. On the other hand, the low energy spectra show no characteristic K- $\alpha$ emissions of iron $(\sim 6.4 \mathrm{keV})$ indicating that their generation is not from the walls of the chamber.

To the best of our knowledge these are the first ever diagnostic measurements on laser plasmas generated by isolated mass limited microparticles with micro-crystallites of random shape. The bremsstrahlung X-ray efficiency for energies greater than $50 \mathrm{keV}$ (for which the transmission through aluminium and glass is $100 \%$ ) is found to be $1.2 \times 10^{-7} \%$, under isotropic emission assumptions. This compares well with the enhanced X-ray emission observed with liquid droplets ${ }^{17}$ which were proven to be about 70 fold larger than the hard $\mathrm{X}$-ray efficiency possible with the conventional solid targets that are routinely used. It should also be kept in mind that most of the earlier experiments from which such references could be drawn are with spherical drops and the experiments are carried out at much larger pulse durations than those used in the current experiments. A detailed study of the physics of plasma generation in the micro-crystalline boric acid particles needs to be elaborated to understand the absorption mechanisms operational here with the shorter pulse. It is possible that the anharmonic resonance heating, a universal mechanism, that is effective with the ellipsoidal E. coli bacteria could play a role here. Arbitrary shapes of the crystallites with sharp features could lead to enhanced local fields and enhanced anharmonicity in the electrostatic potential to lead to enhanced $\mathrm{X}$-ray efficiency. Detailed computations are necessary to elucidate the role of different mechanisms and are currently the efforts towards this end. Further plasma diagnostics need to be applied to understand the micro-plasma generation and evolution. The new delivery mechanism presented here, therefore, offers a unique opportunity to study these effects in detail by systematically varying the size and geometry of particles in the aerosol.

\section{CONCLUSIONS}

In this submission we project that micro/nano-structured targets for laser plasma studies open an exciting prospect both in fundamental understanding and in applications. We propose a mechanism for the delivery of isolated, mass-limited mesoscopic particles for laser plasma which is compact and lends itself adaptable to be used in target vacuum chambers for laser-plasma studies. Enabled by mechanical fluidization, we have shown a repeatable, consistent particle beam for durations of $\geq 30 \mathrm{~min}$, wherein the densities have been measured to be for $15 \mu \mathrm{m}$ particles of boric acid, $\geq 10^{6} \mathrm{~cm}^{-3}$ at the interaction zone. We have also presented here proof of principle 
of the laser plasmas generated in randomly shaped microcrystallite boric acid particles using moderate intensities of $10^{16}$ $\mathrm{W} / \mathrm{cm}^{2}$ and X-ray emission efficiency comparable with the best $^{17}$ studies so far.

With this machine in place, pre-synthesised and sorted mono-disperse particles can be introduced as laser targets and a more systematic study of the intense laser plasma generation can be studied. Experiments with systematic variation of target size would enable us to discern the mechanisms for energy coupling into plasmas at various length scales, hitherto understood only through simulations or using targets embedded on substrates.

In the current version, the laser plasma interaction efficiency is low at $10 \%$, which though sufficient for $\mathrm{kHz}$ repetition rate laser systems is not practical for low repetition rate, often single shot high power laser systems. Partially, this drawback can be addressed by increasing the carrier gas flow, which is however limited by pumping constraints. A further improvement is being explored by us through the inclusion of aerodynamic lenses ${ }^{23}$ to concentrate the particle beam diameter to $0.5 \mathrm{~mm}$ from the current $3 \mathrm{~mm}$. We estimate that this will enable at least an order of magnitude increase in the particle density. Further adaptations to the device to conform to the target chamber for the 100 TW laser facility at Tata Institute of Fundamental Research, Mumbai are also underway to implement experiments at relativistic intensities $\left(10^{18}-10^{19}\right.$ $\left.\mathrm{W} / \mathrm{cm}^{2}\right)$.

\section{ACKNOWLEDGMENTS}

We thank the partial support extended towards this work by the Asian Office of Aerospace R\&D, wide grant: FA238614-1-4023. M.K. thanks the DAR-SRC-OI award for support from the Dept. of Atomic Energy, Government of India.

${ }^{1}$ P. Gibbon and E. Förster, "Short-pulse laser-plasma interactions," Plasma Phys. Controlled Fusion 38(6), 769 (1996).

${ }^{2}$ S. Eliezer, The Interaction of High-Power Lasers with Plasmas (IOP, Bristol, 2002).

${ }^{3}$ C. Thaury, F. Quere, J.-P. Geindre, A. Levy, T. Ceccotti, P. Monot, M. Bougeard, F. Reau, P. d'Oliveira, P. Audebert, R. Marjoribanks, and $\mathrm{Ph}$. Martin, "Plasma mirrors for ultrahigh-intensity optics," Nat. Phys. 4(3), 424-429 (2007).

${ }^{4}$ P. P. Rajeev, P. Taneja, P. Ayyub, A. S. Sandhu, and G. R. Kumar, "Metal nanoplasmas as bright sources of hard x-ray pulses," Phys. Rev. Lett. 90, 115002 (2003).
${ }^{5}$ T. Ditmire, R. A. Smith, J. W. G. Tisch, and M. H. R Hutchinson, "High intensity laser absorption by gases of atomic clusters," Phys. Rev. Lett. 78(16), 3121-3124 (1997).

${ }^{6}$ M. Krishnamurthy, J. Jha, D. Mathur, Ch. Jungreuthmayer, L. Ramunno, J. Zanghellini, and T. Brabec, "Ion charge state distribution in the laser-induced Coulomb explosion of argon clusters," J. Phys. B: At., Mol. Opt. Phys. 39(3), 625 (2006).

${ }^{7}$ T. Ditmire, J. Zweiback, V. P. Yanovsky, T. E. Cowan, G. Hays, and K. B. Wharton, "Nuclear fusion from explosions of femtosecond laserheated deuterium clusters," Nature 398, 489 (1999).

${ }^{8}$ A. Heidenreich, B. Grüner, M. Rometsch, S. R. Krishnan, F. Stienkemeier and M. Mudrich, "Efficiency of dopant-induced ignition of helium nanoplasmas," New J. Phys. 18, 073046 (2016).

${ }^{9}$ A. Mikaberidze, U. Saalmann, and J. M. Rost, "Laser-driven nanoplasmas in doped helium droplets: Local ignition and anisotropic growth," Phys. Rev. Lett. 102, 128102 (2009).

${ }^{10}$ J. Jha, D. Mathur, and M. Krishnamurthy, "Enhancement of x-ray yields from heteronuclear cluster plasmas irradiated by intense laser light," J. Phys. B: At., Mol. Opt. Phys. 38(18), L291 (2005).

${ }^{11}$ S. R. Krishnan, L. Fechner, M. Kremer, V. Sharma, B. Fischer, N. Camus, J. Jha, M. Krishnamurthy, T. Pfeifer, R. Moshammer, J. Ullrich, F. Stienkemeier, M. Mudrich, A. Mikaberidze, U. Saalmann, and J.-M. Rost, "Dopantinduced ignition of helium nanodroplets in intense few-cycle laser pulses," Phys. Rev. Lett. 107, 173402 (2011).

${ }^{12} \mathrm{~S}$. Mondal et al., "Highly enhanced hard x-ray emission from oriented metal nanorod arrays excited by intense femtosecond laser pulses," Phys. Rev. B 83, 035408 (2011).

${ }^{13}$ M. Krishnamurthy, M. Kundu, K. Bane, A. D. Lad, P. Kumar Singh, G. Chatterjee, G. Ravindra Kumar, and K. Ray, "Enhanced x-ray emission from nano-particle doped bacteria," Opt. Express 23, 17909-17922 (2015).

${ }^{14}$ M. Dalui, M. Kundu, T. Madhu Trivikram, R. Rajeev, K. Ray, and M. Krishnamurthy, "Bacterial cells enhance laser driven ion acceleration," Sci. Rep. 4, 6002 (2014).

${ }^{15}$ T. V. Liseykina and D. Bauer, "Plasma-formation dynamics in intense laserdroplet interaction," Phys. Rev. Lett. 110, 145003 (2013).

${ }^{16}$ D. R. Symes, A. J. Comley, and R. A. Smith, "Fast-ion production from short-pulse irradiation of ethanol microdroplets," Phys. Rev. Lett. 93, 145004 (2004).

${ }^{17}$ M. Anand, S. Kahaly, G. R. Kumar, M. Krishnamurthy, A. S. Sandhu, and P. Gibbon, "Enhanced hard x-ray emission from microdroplet preplasma," Appl. Phys. Lett. 88, 181111 (2006).

${ }^{18}$ A. Melling, "Tracer particles and seeding for particle image velocimetry," Meas. Sci. Technol. 8, 1406-1416 (1997).

${ }^{19} \mathrm{~S}$. Ergun and A. A. Orning, "Fluid flow through randomly packed columns and fluidized beds," Ind. Eng. Chem. 41(6), 1179-1184 (1949).

${ }^{20}$ D. Geldart, "Types of gas fluidization," Powder Technol. 7, 285-292 (1973)

${ }^{21}$ J. Visser, "Van der Waals and other cohesive forces affecting powder fluidization," Powder Technol. 58, 1-10 (1989).

${ }^{22}$ E. B. Saloman, J. H. Hubbell, and J. H. Scofield, "X-ray attenuation cross sections for energies $100 \mathrm{eV}$ to $100 \mathrm{keV}$ and elements $\mathrm{Z}=1$ to $\mathrm{Z}=92$," Atomic Data Nucl. Data Tables 38, 1 (1988).

${ }^{23}$ P. Liu, P. J. Ziemann, D. B. Kittelson, and P. H. McMurry, "Generating particle beams of controlled dimensions and divergence: II. Experimental evaluation of particle motion in aerodynamic lenses and nozzle expansions," Aerosol Sci. Technol. 22, 314 (1995). 